In der Rubrik „Literatur kompakt" werden die wichtigsten Originalarbeiten aus der internationalen Fachliteratur referiert.

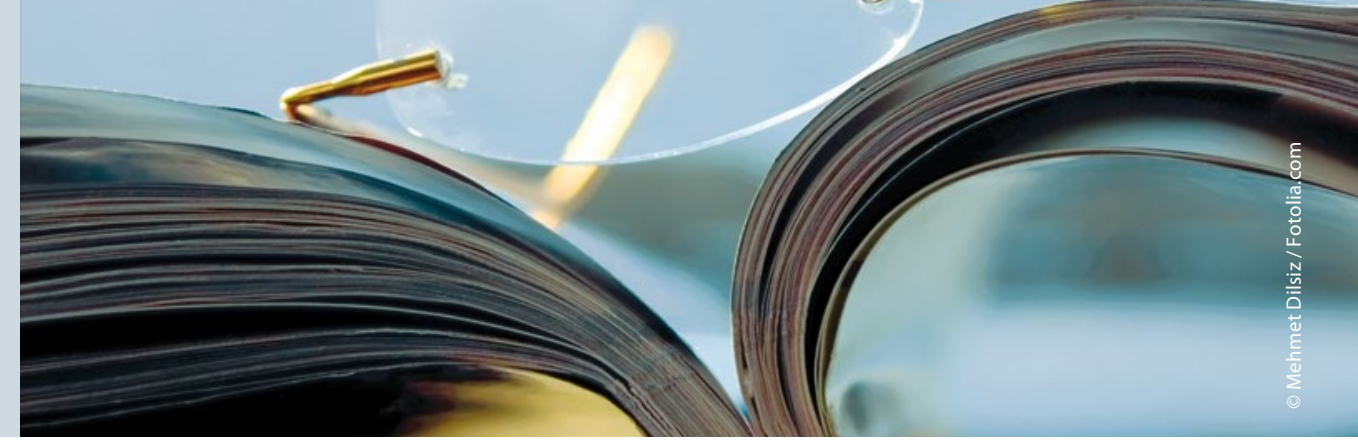

\section{Hypertonie: Sind die Zielwerte für ältere Patienten noch adäquat?}

Die aktuellen Leitlinien empfehlen für Ältere weniger strenge Blutdruckziele als früher. Die vorliegende Arbeit hat eine Gruppe älterer Hypertoniker untersucht, die nach den neuen Empfehlungen nicht mehr zu behandeln sind, nach den älteren Leitlinien hingegen therapiepflichtig waren.

$\mathrm{D}$ ie Studie untersuchte, wie viele Patienten mit relevantem kardiovaskulärem Risiko durch die neuen Empfehlungen unbehandelt bleiben. Untersucht wurde dies bei Patienten, für die früher das Blutdruckziel $<140 / 90 \mathrm{mmHg}$ galt und während der Studie $<150 / 90 \mathrm{mmHg}$. Anhand der Daten aus NHANES 20052012 wurde nach dem FraminghamRisikoscore der Anteil älterer Hypertoniker bestimmt, bei dem das kardiovaskuläre Risiko $\geq 20 \%$ in zehn Jahren betrug und bei dem eine frühere Behandlungsindikation nicht mehr galt.

914 von 12.963 Personen im Alter zwischen 30 und 74 Jahren waren früher behandlungspflichtig und nach den neuen
Empfehlungen nicht. Die Abbildung zeigt den Anteil der Patienten mit einem kardiovaskulären Risiko $\geq 20 \%$ in zehn Jahren. Sie sind unterteilt nach solchen, die unabhängig von den Leitlinien weiterhin behandelt wurden oder unbehandelt waren sowie nach den Begleiterkrankungen chronische Nierenerkrankung und/oder Diabetes mellitus. Unter den behandelten Patienten lag der Anteil mit relevantem kardiovaskulärem Risiko über $50 \%$. Die unbehandelten Hypertoniker hatten in einem etwas geringeren Prozentsatz ein relevantes kardiovaskuläres Risiko. Möglicherweise war somit die Behandlungsentscheidung u.a. von der Einschätzung des kardiovasku-

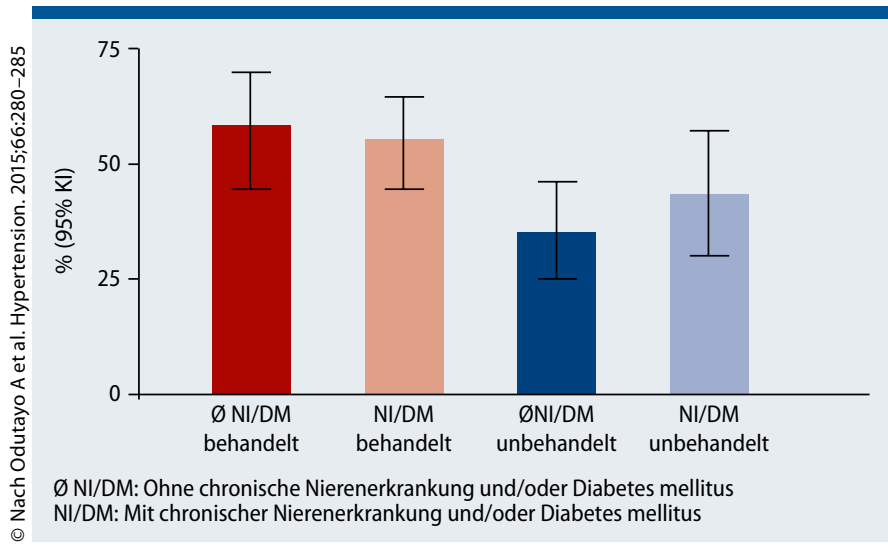

Abb. 1: Anteil der Hypertoniker, die ein kardiovaskuläres Risiko von $\geq 20 \%$ in zehn Jahren haben, früher antihypertensiv zu behandeln waren und nach den neuen JNC8-Leitlinien nicht mehr behandlungspflichtig sind. lären Gesamtrisikos abhängig. Dennoch war bei 34,3 bzw. $42,3 \%$ der unbehandelten Hypertoniker ein relevantes kardiovaskuläres Risiko vorhanden.

Odutayo A et al. Blood Pressure Targets and Absolute Cardiovascular Risk. Hypertension. 2015; 66:280-285.

\section{Kommentar}

Die Arbeit wirft die Frage auf, ob die weniger strenge Blutdruckeinstellung auch für ältere Hypertoniker mit höherem kardiovaskulärem Risiko adäquat ist. Bereits bei der Veröffentlichung der US-amerikanischen Leitlinien (JNC8) wurde parallel ein „Minderheitenvotum" publiziert, das auf die Nachteile einer weniger strengen Blutdruckeinstellung hinwies. Ein kardiovaskuläres Risiko von $\geq 20 \%$ über zehn Jahre gilt als hohes Risiko. Diese Patienten sind nach den neuesten ESH/ESC-Leitlinien medikamentös zu behandeln mit einem Blutdruckziel $<140 / 90$ mmHg. Für ca. 30 - $40 \%$ der Hypertoniker zwischen 60 und 74 Jahren sind also die Empfehlungen möglicherweise widersprüchlich, insofern als das kardiovaskuläre Gesamtrisiko einen niedrigeren Zielwert als 150/90 mmHg erfordern würde. Die Arbeit zeigt somit erneut, dass die Diskussion über die optimale Blutdrucksenkung speziell beim älteren Patientenkollektiv nicht abgeschlossen ist und nach wie vor die individuelle klinische Einschätzung bei der Therapieeinstellung einen hohen Stellenwert hat.

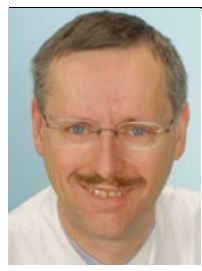

Prof. Dr. med. Walter Zidek

Medizinische Klinik IV, Charité Universitätsmedizin Berlin 